\title{
Medium-chain triglycerides and conjugated linoleic acids in beverage form increase satiety and reduce food intake in humans
}

Article

Accepted Version

Creative Commons: Attribution-Noncommercial-No Derivative Works 4.0

Coleman, H., Quinn, P. and Clegg, M. E. (2016) Medium-chain triglycerides and conjugated linoleic acids in beverage form increase satiety and reduce food intake in humans. Nutrition Research, 36 (6). pp. 526-533. ISSN 1879-0739 doi: https://doi.org/10.1016/j.nutres.2016.01.004 Available at https://centaur.reading.ac.uk/80391/

It is advisable to refer to the publisher's version if you intend to cite from the work. See Guidance on citing.

Published version at: http://www.ncbi.nlm.nih.gov/pubmed/27188898

To link to this article DOI: http://dx.doi.org/10.1016/j.nutres.2016.01.004

Publisher: Elsevier

All outputs in CentAUR are protected by Intellectual Property Rights law, including copyright law. Copyright and IPR is retained by the creators or other copyright holders. Terms and conditions for use of this material are defined in the End User Agreement. 


\section{CentAUR}

Central Archive at the University of Reading

Reading's research outputs online 
1 Medium chain triglycerides and conjugated linoleic acids in beverage form increase

2 satiety and reduce food intake in humans.

3

4 Hannah Coleman, Paul Quinn and Miriam E Clegg*

5

6 Functional Food Centre, Oxford Brookes University, Gipsy lane, Headington, Oxford OX3

7 0BP, United Kingdom

8

$9 \quad *$ Corresponding author:

10 Email: mclegg@brookes.ac.uk

11 Ph: +44 1865484365

12

13

14

15

16

17

18

19

20

21

22

23

24

25 
List of abbreviations

27

28 CLA - conjugated linoleic acid

29 MCT - medium chain triglycerides

30 VAS - visual analogue scales

31

32

33

34

35

36

37

38

39

40

41

42

43

44

45

46

47

48

49

50 
Both developed and developing countries are seeing increasing trends of obesity in people young and old. It is thought satiety may play a role in the prevention of obesity by increasing satiety and reducing energy intake. We hypothesized that medium chain triglycerides (MCT) would increase satiety and decrease food intake compared to conjugated linoleic acid (CLA) and a control oil. 19 healthy participants were tested on three separate occasions, where they consumed a beverage test breakfast containing either (1) vegetable oil (control) (2) CLA or (3) MCT. Participants self-requested an ad libitum sandwich buffet lunch. Time between meals, satiety from visual analogue scales (VAS), energy intake at lunch, and intake for the rest of the day using weighed food diaries were measured. The results indicated that the time until a meal request was significantly different between the three meals $(p=0.016)$, however there were no differences in intakes at the ad libitum lunch $(\mathrm{p}>0.05)$. The CLA breakfast generated the greatest delay in meal time request. There was a difference between the control lipid compared to both the CLA and MCT for energy intake over the remainder of the test day and for total energy intake on the test day ( $<<0.001$ for both), with the CLA and MCT resulting in a lower intake than the control throughout the day. There were no significant differences in satiety from VAS scores ( $>>0.05)$. Both CLA and MCT increased satiety and reduced energy intake, indicating a potential role in aiding the maintenance of energy balance.

Keywords: Medium chain triglycerides; conjugated linoleic acid; satiety; food intake 


\section{Introduction}

77

78

According to the World Health Organisation [1], obesity has nearly doubled worldwide since 1980 and obesity rates in both men and women have increased by over $10 \%$ in the UK alone since 1993 [2]. Obesity can develop into a major health problem increasing the risk of developing numerous diseases, including type II diabetes, cardiovascular disease and premature death [3]. The leading causes of obesity are lack of physical activity and overconsumption of high energy food [4]. With individuals and governments searching for different solutions to weight loss and fat reduction, the use of dietary supplements has increased significantly in recent years [5] [6]. It is possible that satiety may play a key role in the development of obesity [7].

Benelam [8] defines satiety as "the feeling of fullness that persists after eating, potentially suppressing further energy intake until hunger returns". It is possible that increasing satiety, and thus, delaying the onset of food intake can lead to less food intake at the next meal and throughout the rest of the day. If food intake is lowered then the risk of obesity will potentially reduce. It is possible that certain foods can play a role in increasing satiety, and thus, reduce overall food intake. At the very least they may encourage individuals to be less distracted by cues to consume, and enable them to maintain regular eating habits [9].

Conjugated linoleic acid (CLA) refers to a class of positional and geometric conjugated dienoic isomers of linoleic acid that is naturally present in the meat of ruminants. Cis- 9 , trans-11 CLA and trans-10, cis-12 make up the main isomers of CLA [10]. CLA is believed to have a positive effect on human health, particularly on body weight and body fat [11]. It is thought that the isomer trans-10, cis-12 is responsible for positive changes in body 
composition [10]. Some studies have shown that daily intake of CLA can reduce both body weight and body fat [12-14] though the clinical relevance of these changes is still open to debate [15]. There has been little research conducted on the effects of CLA on satiety. Several studies have assessed the effect of CLA on appetite [16-18]. However this research has primarily focused on subjective ratings of appetite or following a CLA intervention. To the authors knowledge no data has examined the effect on actual food intake during a one day trial. In rats however, some studies found decreased energy intakes following CLA consumption [19-21] whereas other studies observed no effect on food intake [22-24]. It is known that intake of CLA decreases the uptake of fatty acids into adipocytes and increases $\beta$ oxidation in muscle cells. A potential theory is that this may result in a shift towards fat oxidation that could result in glycogen being spared. This may in turn serve as a satiety signal, as has been proposed by several researchers [25, 26]. However this mechanism has not been proved in other research $[27,28]$ so speculation as to how CLA can increase satiety remains open to debate. Studies on medium-chain triglycerides have been much more frequent.

Medium-chain triglycerides (MCTs) are triglycerides with a fatty acid chain length varying between 6 and 10 carbon atoms. MCTs are soluble in water, rapidly absorbed and preferentially oxidised compared to long-chain triglycerides (LCTs). The most common sources of MCTs are coconut oil, palm oil and dairy fat; however it is most commonly used as a weight loss aid in the form of synthetic oil [29] where over 16 weeks it has been shown to result in greater weight losses than olive oil $(-1.67+/-0.67 \mathrm{~kg})[30]$. MCT has demonstrated it's ability to increase satiety by delaying meal requests and reducing food intake by up to $698 \mathrm{~kJ}$ compared to a saturated lipid [31]. MCTs ability to increase satiety is believed to be due to its increased oxidative capacity; however the exact mechanisms are 
unknown. MCTs undergo nearly complete hydrolysis to free fatty acids (FFA) after ingestion,

127

128 and are then absorbed directly into the portal vein. Then they are transported rapidly to the liver for $\beta$-oxidation. LCTs, differ however, as they are absorbed via the intestinal lymphatic ducts at a much slower rate and transported by chylomicrons into the systemic circulation prior to oxidation or storage. MCTs are faster oxidised than LCTs [32]. Therefore they are a much more readily available energy source. Several studies have been unable to detect differences in satiety following MCT [33, 34], however other studies have shown MCT's can be beneficial to increasing satiety and reducing energy intake, and thus, causing weight loss [35]. However, how different lipids compare in terms of their ability to increase satiety is less well known.

The objectives of this study were twofold. Firstly, to examine the effect of CLA on satiety and food intake. To the author's knowledge this has not previously been completed in a single day trial assessing food intake and subjective satiety. The second objective of this study was to compare the effect of CLA to MCT in terms of satiety and food intake. The authors hypothesize that MCT will increase satiety more than CLA or a control lipid. The methods used to measure satiety in this study included a self-requested ad libitum buffet lunch and visual analogue scales.

\section{Methods and materials}

\subsection{Participants}

Participants were recruited through the use of posters, social networking and word of mouth. Prior to participation all participants were tested for suitability through both a pre-test 
questionnaire and a dietary restraint questionnaire [36]. Twenty-six participants were recruited in total. Eating behaviour was determined using the Dutch eating behaviour questionnaire [37]. Only those who did not consciously restrain their food intake due to psychological reasons, weight concerns and external stimuli were included in the study. Those who fulfilled all the acceptable criteria (age 18-60 years; body mass index $<30 \mathrm{~kg} / \mathrm{m}^{2}$; blood pressure 110-120/75-85 mmHg; non-smoking; not highly physically active or involved in sports at the endurance and competitive levels ( $>10$ hours a week vigorous exercise); not suffering from any eating disorders; not allergic/intolerant to any of the foods presented in the study; habitually consuming breakfast and lunch; not on prescription medication; no genetic or metabolic diseases) were included in the study. On the day before each test, participants were asked to restrict their intake of alcohol and caffeine containing drinks and to refrain from strenuous physical activity.

All participants were given an information sheet explaining the study and the possible risks to taking part prior to giving informed consent. Ethical approval was granted by the Research Ethics officer at Oxford Brookes University in line with the Declaration of Helsinki.

Participants were asked to fast for 12 hours prior to testing and to not do any strenuous exercise the morning of the test. 19 participants (12f; $31.4 \pm 18.0 \mathrm{yr} ; 169 \pm 11 \mathrm{~cm} ; 68.6 \pm$ $11.7 \mathrm{~kg}$ ) completed the study (figure 1).

Participants took part in a randomised, single blind study. Participants were required to attend the laboratory from $9 \mathrm{am}$ to $2 \mathrm{pm}$ on three separate non-consecutive days. Participants consumed a test breakfast containing CLA, MCT or a control oil (vegetable oil), following which their appetite and satiety were monitored. Prior to the first test participants recorded 
the previous day's food intake using a weighed food diary and repeated this food intake on the day prior to the subsequent tests.

\subsection{Breakfast}

The test breakfast consisted of $250 \mathrm{ml}$ of Tesco red berries smoothie $-123 \mathrm{kcal}(515 \mathrm{~kJ}), 0.8 \mathrm{~g}$ protein, $29.8 \mathrm{~g}$ carbohydrate, $27.0 \mathrm{~g}$ sugar and $0 \mathrm{~g}$ fat. Added to it was $193 \mathrm{kcal}(808 \mathrm{~kJ})$ of lipids, consisting of either $5 \mathrm{~g}$ CLA (Trec Nutrition, London, UK) and $16 \mathrm{~g}$ vegetable oil, $25 \mathrm{~g}$ MCT (Trec Nutrition, London, UK) or $22 \mathrm{~g}$ vegetable oil as a control (Tesco, Cheshunt, UK). All lipids were added in these doses so that smoothies had the same energy and fat content. The total energy content of each smoothie was $316 \mathrm{kcal}(1323 \mathrm{~kJ})$. Doses used for each lipid was based on previous studies, considered safe and sufficient enough to see a possible effect $[14,38]$. Pretesting was undertaken to ensure that the three drinks tasted similar and palatable.

\subsection{Subjective satiety}

Satiety was measured using visual analogue scales (VAS). Participants were asked to fill out a 100mm VAS before and after the test breakfast. The VAS were anchored at the left and right ends with opposing statements for feelings of hunger, fullness, desire to eat and prospective food consumption. The specific questions asked were, 'How hungry do you feel?', 'How full do you feel?', 'How strong is your desire to eat?' and 'How much food do you think you can eat?'. The VAS contained numbers ranging from 1-10, with 1 being low and 10 being high. The VAS were completed by participants every half hour after the breakfast up until the participant felt hungry enough to request lunch. The time taken between breakfast and the request for lunch was measured for each participant as previously 
undertaken by Van Wymelbeke et al [31]. Because the participants requested their lunches and dinners at different times, the scores given in the VAS were analysed up to 60 minutes as this was the time at which the first person requested their lunch. This was the method previously used by Van Wylebeke et al [31]. Participants were allowed 500ml of water during the time between breakfast and lunch on the first test. This was measured and repeated in subsequent tests.

\subsection{Food intake}

Participants were asked to let the researchers know when they felt hungry enough to eat lunch following the test breakfast. Participant had to stay in the laboratory until $2 \mathrm{pm}$ regardless of how soon they requested their ad libitum lunch. All time cues were removed from the participants view - clocks on laptops were covered with paper and tape and phones and watches were removed. Once lunch was requested, sandwiches were given ad-libitum to measure food intake similar to that used by Ranawana et al [39] and Clegg and Thondre [40]. Prior to testing participants were given a choice of sandwiches from a list prior to testing and asked to choose which ones they liked. All the sandwich recipes were formulated to contain the same energy content per portion (Table 1). The lunch consisted of three weighed plates each containing two sandwiches cut into quarters. Participants were given all the sandwiches at once so that it was in excess and asked to eat until they felt comfortably full. Participants were given the same sandwiches for each test. The subjects were presented with the meal under identical conditions on each test day. They ate in the same laboratory on their own with no distractions and were given 30 minutes in which to eat their ad libitum meal. 
When participants finished eating and the remaining food leftover was weighed to measure food intake. A food diary was used to measure food intake for the rest of the day. Food diaries were analysed using the software package Nutritics Professional (Est. 2011, Dublin, Ireland).

\subsection{Statistical analyses}

Statistical analyses was performed using Statistical Package for the Social Sciences (version 20.0; SPSS, Chicago, IL, USA) and data and figures were processed using Microsoft Excel (2006, Reading, UK). A power calculation was conducted for the primary outcome measure of energy intake. A sample size of 19 was required to detect a $300 \mathrm{~kJ}$ difference in energy intake with a standard deviation of $250 \mathrm{~kJ}$ and $\alpha$ set at 0.05 and a power of $90 \%$ [31].

A repeated measures ANOVA with Bonferroni correction was performed on the food intake and time-to-meal-request data to gage if there were any significant differences in satiety levels between lipids. A repeated measures ANCOVA was used for analysis of the VAS data up to and including the 60 minute data. The baseline was used as a covariate in the analysis. The significance value was set at $\mathrm{p}<0.05$.

\section{Results}

\subsection{Ad libitum lunch}

For the ad libitum lunch (Table 2) there were no significant differences in intake between the control, CLA or MCT tests on energy or any macronutrients ( $p>0.05)$. Energy and 
macronutrient intake was highest amongst the control group, an average of $70 \mathrm{kcal}(293 \mathrm{~kJ})$ more than CLA and MCT. Macronutrient intake was similar after consumption of both CLA and MCT at lunch.

\subsection{Rest of day intake}

There were significant differences in food intake from the rest of the day (Table 2) following the ad libitum lunch between the three meals $(\mathrm{p}<0.001)$. These differences were found between the MCT meal and the control, and between the CLA meal and the control. The MCT breakfast resulted in the least amount of energy consumed after lunch and the control had the highest intake, with an average of $471 \mathrm{kcal}(1972 \mathrm{~kJ})$ more consumed following the control compared to CLA and $525 \mathrm{kcal}(2198 \mathrm{~kJ})$ more compared to MCT. There were also significant differences in intake of all macronutrients following the three breakfasts (protein $\mathrm{p}=0.003$, fat $\mathrm{p}<0.001$; carbohydrate $\mathrm{p}<0.001$ ). These differences were found between the two test lipids and the control for all three macronutrients with the exception of protein, in which the CLA was not significantly different to the control.

\subsection{Total days intake}

The results showed that having the control breakfast resulted in the greatest energy intake, an average of $541 \mathrm{kcal}(2265 \mathrm{~kJ})$ more compared to CLA and $594 \mathrm{kcal}(2487 \mathrm{~kJ})$ more than MCT $(p<0.001)$. There were significant differences following the control compared to CLA and MCT for total energy intake and on all macronutrient intakes with the exception of protein between the control and the CLA breakfast. The MCT showed the greatest satiating effect. 
The time until a meal request (Table 3) showed that there was a significant difference in the time until lunch was requested between the three meals $(\mathrm{p}=0.016)$. These differences existed between the control breakfast and the CLA breakfast $(\mathrm{p}=0.049)$. The control delayed the meal request the least, followed by the MCT with the CLA delaying the time until lunch the most.

\subsection{Visual analogue scale}

There were no significant differences between any of the three tests on any of the four questions hunger, fullness, desire to eat or prospective consumption ( $p>0.05$; Figure 2$)$. Perceived satiety increased immediately following the breakfast and then decreased again at 30 and 60 minute.

\section{Discussion}

291

As far as the authors of this study are aware, this is the first study that has compared the effect of both CLA and MCTs on food intake and satiety within the same study. The results from this study show that both CLA and MCTs reduce and delay food intake over a day when compared to a control. The results showed that there were significant differences in time to lunch request, energy and macronutrient intakes for the rest of the day (after test breakfast and ad libitum lunch) and over the entire day between the three meals. These differences were seen between the CLA and MCT compared to the control. There were no significant differences between CLA and MCT intakes at any stage or for any parameter. These results 
show that both test lipids increased satiety and thus reduced energy intake hence rejecting the original hypothesis that only MCT would increase satiety above the control.

Data on CLA and satiety is limited. One previous study [18] found that CLA did not reduce ad libitum energy intake during breakfast after consuming a dose of either $1.8 \mathrm{~g}$ or $3.6 \mathrm{~g}$ per day and after an overnight fast, though feelings of fullness and satiety were increased and feelings of hunger were decreased compared to placebo. However, this study was looking at the effect of a 13 week CLA intervention and CLA had not been consumed since the day before. This may indicate that the results are due to long term dietary changes rather than the effect of a single dose of CLA. A similar study conducted by Lambert et al. [17] found no significant reduction in subjective satiety ratings after a standardised breakfast following CLA supplementation for 12 weeks. The current study was able to demonstrate the effect of a single dose of CLA on meal request and food intake.

In contrast to CLA the short term effects of MCTs are well documented, showing a decreased intake at lunch following an MCT rich breakfast $[31,41]$. However the current study did not find a difference in food intake at lunch or a significant delay in the meal request, though this did approach significance. It is possible taking a dose of at least $25 \mathrm{~g}$ of MCTs in the morning can reduce energy intake later in the day i.e. after lunch. This result is similar to that in other studies where intake of MCT reduced energy intake later in the day [42], and research that showed that food intake at dinner was reduced following an MCT lunch but similar to the current study the meal request was not delayed [43]. Interestingly the current study was not able to detect any difference in satiety between the two test lipids indicating that they both were equally as satiating as the other. 
For the ad libitum lunch, there were no significant differences in intakes between CLA, MCT or the control. However this may be due to the meal request being earlier following the CLA and MCT and may indicate that the participants were truly able to detect their level of hunger and accurately compensate for this. The methods used were chosen to test the possibility that the test lipids allow a longer time period between breakfast and lunch. Had participants been given lunch at a set time, it would not have allowed the possibility to test the duration of satiety. It was decided that participants would be taken into the laboratory in the morning, given the test breakfast, and sent back out to a waiting area. There was no set time for lunch and participants were told to tell the researchers when they felt hungry enough to eat lunch, which worked efficiently in a previous study [31]. Participants were told they were to be in the laboratory from 9 a.m. to 2 p.m. This eliminated the temptation to request lunch in order to be able to leave the laboratory prior to feeling genuine hunger. If participants finished lunch prior to 2 p.m. they were asked to wait in the laboratory until then. Participants were asked to fill out food diaries for the rest of the day after the ad libitum lunch. Although this was aimed to replicate a free -living element to the study it is known that people often underestimate their food intake when filling out food diaries or don't eat as they normally would as they know they are recording it [44] which could impact on the results.

Nausea, stomach cramping and other gastrointestinal problems are a known side effect of MCTs [35]. Five participants in the present study reported suffering side effects of this nature after ingesting the MCT breakfast. This shows that even a dose as small as $25 \mathrm{~g}$ of MCTs can have side effects which may have impacted in their food intake. One participant suffered gastrointestinal discomfort from ingesting the CLA. There were no side effects from ingesting the control. 
There were several limitations to this study. The use of food diaries as highlighted previously does not control the environment in which the participant is tested, however they do have their merits including high external validity and applicability to real life situations [45]. The two test lipids were not matched in terms of energy content due to a compromise between seeing positive results and not having adverse effects on the participants. Instead doses were chosen based on previous literature. Although pretesting was completed in a different population, no direct measures of palatability were completed in the current volunteers so they would not be concerned about differences between the beverages. However there is a chance that the current cohort of volunteers was able to detect differences that might have influenced their palatability and intake. GI disturbances were not recorded during the study but the participant was asked about these after each day so as not to influence their thoughts on this issue. Finally each individual had the same sandwiches for all three of their ad libitum meals which may have become monotonous and caused sensory specific satiety however the volunteers were given sandwiches to suit their preferences. Johnson and Vickers[46] have previously outlined that there is a trend for less-liked test meals to drop more in liking than the well-liked test meals following repeated exposure.

The present study reveals that both CLA and MCT can increase satiety and decrease food intake over a period of a day. Given the side effects seen following MCT consumption and that CLA consumption resulted in had similar satiating effects, CLA may be proposed as an alternative food ingredient to increase satiety. This may be beneficial to future prevention and/or treatment of obesity; however more research is needed including longer duration laboratory trials particularly on CLA and its effect on satiety and food intake. 
375 Acknowledgement:

376 This research was funded by Oxford Brookes University. No product or funding was received

377 from Trec Nutrition.

378

379

380

381

382

383

384

385

386

387

388

389

390

391

392

393

394

395

396

397

398

399

400

401 
[1] World Health Organisation. Obesity and Overweight. 2014 [cited 2015 13th January]; Available from: http://www.who.int/mediacentre/factsheets/fs311/en/

[2] Lifestyles statistics team HaSCIC (2014) Statistics on Obesity, Physical Activity and Diet England

[3] Wellman NS, Friedberg B. Causes and consequences of adult obesity: health, social and economic impacts in the United States. Asia Pac J Clin Nutr 2002;11 Suppl 8:S705-9.

[4] National Institutes of Health. Causes Overweight and Obesity. 2012 [cited 2014 13th January]; Available from: http://www.nhlbi.nih.gov/health/healthtopics/topics/obe/causes.html

[5] Johansson K, Neovius M, Hemmingsson E. Effects of anti-obesity drugs, diet, and exercise on weight-loss maintenance after a very-low-calorie diet or low-calorie diet: a systematic review and meta-analysis of randomized controlled trials. Am J Clin Nutr 2014;99(1):14-23.

[6] Jeukendrup AE, Randell R. Fat burners: nutrition supplements that increase fat metabolism. Obesity Reviews 2011;12(10):841-851.

Halford JC, Harrold JA. Satiety-enhancing products for appetite control: science and regulation of functional foods for weight management. Proc Nutr Soc 2012;71(2):350-62.

[9] Hetherington MM, Cunningham K, Dye L, Gibson EL, Gregersen NT, Halford JC et al. Potential benefits of satiety to the consumer: scientific considerations. Nutr Res Rev 2012;26(1):22-38 composition. J Am Diet Assoc 2004;104(6):963-8, quiz 1032. 
[12] Gaullier JM, Halse J, Hoye K, Kristiansen K, Fagertun H, Vik H et al. Supplementation with conjugated linoleic acid for $\mathbf{2 4}$ months is well tolerated by and reduces body fat mass in healthy, overweight humans. J Nutr 2005;135(4):778-84.

[13] Chen SC, Lin YH, Huang HP, Hsu WL, Houng JY, and Huang CK. Effect of conjugated linoleic

[14] Blankson H, Stakkestad JA, Fagertun H, Thom E, Wadstein J, and Gudmundsen O. Conjugated acid supplementation on weight loss and body fat composition in a Chinese population. Nutrition 2012;28(5):559-65. linoleic acid reduces body fat mass in overweight and obese humans. J Nutr 2000;130(12):2943-8.

[15] Onakpoya IJ, Posadzki PP, Watson LK, Davies LA, Ernst E. The efficacy of long-term conjugated linoleic acid (CLA) supplementation on body composition in overweight and obese individuals: a systematic review and meta-analysis of randomized clinical trials. Eur J Nutr 2012;51(2):127-34.

[16] Medina EA, Horn WF, Keim NL, Havel PJ, Benito P, Kelley DS et al. Conjugated linoleic acid supplementation in humans: effects on circulating leptin concentrations and appetite. Lipids 2000;35(7):783-8.

[17] Lambert EV, Goedecke JH, Bluett K, Heggie K, Claassen A, Rae DE et al. Conjugated linoleic acid versus high-oleic acid sunflower oil: effects on energy metabolism, glucose tolerance, blood lipids, appetite and body composition in regularly exercising individuals. Br J Nutr 2007;97(5):1001-11.

[18] Kamphuis MM, Lejeune MP, Saris WH, Westerterp-Plantenga MS. Effect of conjugated linoleic acid supplementation after weight loss on appetite and food intake in overweight subjects. Eur J Clin Nutr 2003;57(10):1268-74.

[19] Miner JL, Cederberg CA, Nielsen MK, Chen X, Baile CA. Conjugated linoleic acid (CLA), body fat, and apoptosis. Obes Res 2001;9(2):129-34. 
[20] Park Y, Albright KJ, Storkson JM, Liu W, Cook ME, and Pariza MW. Changes in body composition in mice during feeding and withdrawal of conjugated linoleic acid. Lipids $1999 ; 34(3): 243-8$.

[21] West DB, Delany JP, Camet PM, Blohm F, Truett AA, and Scimeca J. Effects of conjugated linoleic acid on body fat and energy metabolism in the mouse. Am J Physiol 1998;275(3 Pt 2):R667-72.

[22] Azain MJ, Hausman DB, Sisk MB, Flatt WP, Jewell DE. Dietary conjugated linoleic acid reduces rat adipose tissue cell size rather than cell number. J Nutr 2000;130(6):1548-54.

[23] DeLany JP, Blohm F, Truett AA, Scimeca JA, West DB. Conjugated linoleic acid rapidly reduces body fat content in mice without affecting energy intake. Am J Physiol 1999;276(4 Pt 2):R1172-9.

[24] Sisk MB, Hausman DB, Martin RJ, Azain MJ. Dietary conjugated linoleic acid reduces adiposity in lean but not obese Zucker rats. J Nutr 2001;131(6):1668-74.

[25] Flatt JP. Glycogen levels and obesity. Int J Obes Relat Metab Disord 1996;20 Suppl 2:S1-11.

[26] Melanson KJ, Westerterp-Plantenga MS, Campfield LA, Saris WH. Appetite and blood glucose profiles in humans after glycogen-depleting exercise. J Appl Physiol (1985) 1999;87(3):94754.

[27] Shetty PS, Prentice AM, Goldberg GR, Murgatroyd PR, McKenna AP, Stubbs RJ et al. Alterations in fuel selection and voluntary food intake in response to isoenergetic manipulation of glycogen stores in humans. Am J Clin Nutr 1994;60(4):534-43.

[28] Stubbs RJ, Murgatroyd PR, Goldberg GR, Prentice AM. Carbohydrate balance and the regulation of day-to-day food intake in humans. Am J Clin Nutr 1993;57(6):897-903.

[29] Marten B, Pfeuffer M, Schrezenmeir J. Medium-chain triglycerides. International Dairy Journal 2006;16(11):1374-1382. 
[30] St-Onge MP, Bosarge A. Weight-loss diet that includes consumption of medium-chain triacylglycerol oil leads to a greater rate of weight and fat mass loss than does olive oil. Am J Clin Nutr 2008;87(3):621-6.

[31] Van Wymelbeke V, Himaya A, Louis-Sylvestre J, Fantino M. Influence of medium-chain and long-chain triacylglycerols on the control of food intake in men. Am J Clin Nutr $1998 ; 68(2): 226-34$

[32] Dulloo AG, Fathi M, Mensi N, Girardier L. Twenty-four-hour energy expenditure and urinary catecholamines of humans consuming low-to-moderate amounts of medium-chain triglycerides: a dose-response study in a human respiratory chamber. Eur J Clin Nutr 1996;50(3):152-8.

[33] Bendixen H, Flint A, Raben A, Hoy CE, Mu H, Xu X et al. Effect of 3 modified fats and a conventional fat on appetite, energy intake, energy expenditure, and substrate oxidation in healthy men. Am J Clin Nutr 2002;75(1):47-56.

[34] Poppitt SD, Strik CM, MacGibbon AK, McArdle BH, Budgett SC, and McGill AT. Fatty acid chain length, postprandial satiety and food intake in lean men. Physiol Behav 2010;101(1):161-7.

[35] Clegg ME. Medium-chain triglycerides are advantageous in promoting weight loss although not beneficial to exercise performance. Int J Food Sci Nutr 2010.

[36] Urbszat D, Herman CP, Polivy J. Eat, drink, and be merry, for tomorrow we diet: effects of anticipated deprivation on food intake in restrained and unrestrained eaters. J Abnorm Psychol 2002;111(2):396-401.

[37] van Strien T, Frijter JER, Bergers GPA, Defares PB. The Dutch Eating Behaviour Questionnaire (DEBQ) assessment of restrained, emotional, and external eating behavior. Int J Eat Disord $1986 ; 5(2): 295-315$. 
[38] Clegg ME, Golsorkhi M, Henry CJ. Combined medium-chain triglyceride and chilli feeding increases diet-induced thermogenesis in normal-weight humans. Eur J Nutr 2013;52(6):1579-85.

[39] Ranawana V, Muller A, Henry CJ. Polydextrose: its impact on short-term food intake and subjective feelings of satiety in males-a randomized controlled cross-over study. Eur J Nutr;52(3):885-93.

[40] Clegg ME, Thondre PS. Molecular weight of barley beta-glucan does not influence satiety or energy intake in healthy male subjects. Appetite 2014;83:167-72.

[41] Rolls BJ, Gnizak N, Summerfelt A, Laster L. Food intake in dieters and nondieters after a liquid meal containing medium-chain triglycerides. Am J Clin Nutr 1988;48(1):66-71.

[42] Stubbs RJ, Harbron CG. Covert manipulation of the ratio of medium- to long-chain triglycerides in isoenergetically dense diets: effect on food intake in ad libitum feeding men. Int J Obes Relat Metab Disord 1996;20(5):435-44.

[43] Van Wymelbeke V, Louis-Sylvestre J, Fantino M. Substrate oxidation and control of food intake in men after a fat-substitute meal compared with meals supplemented with an isoenergetic load of carbohydrate, long-chain triacylglycerols, or medium-chain triacylglycerols. Am J Clin Nutr 2001;74(5):620-30.

[44] Black AE, Prentice AM, Goldberg GR, Jebb SA, Bingham SA, Livingstone MB et al. Measurements of total energy expenditure provide insights into the validity of dietary measurements of energy intake. J Am Diet Assoc 1993;93(5):572-9.

[45] Blundell J, de Graaf C, Hulshof T, Jebb S, Livingstone B, Lluch A et al. Appetite control: methodological aspects of the evaluation of foods. Obes Rev 2010;11(3):251-70.

[46] Johnson J, Vickers Z. Effects of flavor and macronutrient composition of food servings on liking, hunger and subsequent intake. Appetite 1993;21(1):25-39. 
528 Figure 1: Flow chart showing participant recruitment

529

530

531

532

533

534

535

536

537

538

539

540

19 participants completed the study

541

542

543

544

545

546

547

548

549

550

551

552 
553 Figure 2: Visual analogue scale data for hunger, fullness, desire to eat and prospective

554 consumption at baseline ( 0 min), after breakfast (post break), and 30 and 60 min after

555 breakfast $^{\text {a }}$.

556

$557 \quad{ }^{a}$ Values are means $\pm \mathrm{SD}$

558

559

560

561

562

563

564

565

566

567

568

569

570

571

572

573

574

575

576

577 
578 Table 1: Nutritional content of sandwiches (ad libitum lunch)

\begin{tabular}{|c|c|c|c|c|c|}
\hline Sandwich: & Weight (g) & Energy $(\mathrm{kcal}(\mathrm{kJ}))$ & Carbohydrate (g) & Protein (g) & Fat $(g)$ \\
\hline Egg mayo & 223 & 408.20 (1709) & 36.68 & 17.46 & 19.81 \\
\hline \multicolumn{6}{|l|}{ Cheese and } \\
\hline tomato & 185 & $406.06(1700)$ & 36.62 & 19.73 & 18.51 \\
\hline Tuna mayo & 146 & $402.79(1686)$ & 35.30 & 18.37 & 19.56 \\
\hline Chicken salad & 221 & $406.48(1701)$ & 37.51 & 18.61 & 18.66 \\
\hline \multicolumn{6}{|l|}{ Cheese and } \\
\hline pickle & 148 & $404.75(1695)$ & 38.98 & 19.03 & 17.75 \\
\hline \multicolumn{6}{|l|}{ Ham and } \\
\hline cheese & 153 & 405.43 (1698) & 35.62 & 21.49 & 18.21 \\
\hline \multicolumn{6}{|l|}{ Roast beef and } \\
\hline tomato & 181 & $404.30(1693)$ & 36.55 & 20.02 & 18.11 \\
\hline
\end{tabular}

579

$580{ }^{a}$ Three plates of sandwiches were served at each ad libitum lunch

581

582

583

584

585

586

587

588

589

590 
591 Table 2: Energy and macronutrient intake at the ad libitum lunch, for the rest of the day

592 following the lunch and the day's total intake ${ }^{a, b, c}$

\begin{tabular}{lccc}
\hline & Control & CLA & MCT \\
\hline \multicolumn{3}{c}{ Ad Libitum Lunch } \\
\hline Energy (kcal) & $798.45 \pm 207.91$ & $728.61 \pm 188.38$ & $728.73 \pm 182.91$ \\
$\mathrm{~kJ}$ & $3343 \pm 870$ & $3051 \pm 789$ & $3051 \pm 766$ \\
Carbohydrate (g) & $71.87 \pm 19.27$ & $65.38 \pm 16.51$ & $65.40 \pm 16.12$ \\
& & $34.43 \pm 8.53$ & $34.48 \pm 8.51$ \\
Protein (g) & $37.90 \pm 10.24$ & $33.73 \pm 9.45$ & $33.72 \pm 9.06$ \\
Fat $(\mathrm{g})$ & $36.73 \pm 9.36$ & & \\
\hline
\end{tabular}

Rest of day intake

\begin{tabular}{lccc}
\hline Energy (kcal) & $1171.63 \pm 458.36$ & $699.95 \pm 321.49^{*}$ & $646.74 \pm 313.75^{*}$ \\
kJ & $4905 \pm 1919$ & $2931 \pm 1346$ & $2708 \pm 1314$ \\
Carbohydrate $(\mathrm{g})$ & $124.32 \pm 60.48$ & $69.74 \pm 42.60^{*}$ & $64.08 \pm 41.56^{*}$ \\
Protein $(\mathrm{g})$ & $65.05 \pm 30.41$ & $47.79 \pm 23.01$ & $44.49 \pm 20.55^{*}$ \\
Fat $(\mathrm{g})$ & $39.85 \pm 16.82$ & $23.63 \pm 13.76^{*}$ & $21.68 \pm 13.04^{*}$ \\
\hline
\end{tabular}

Total days intake

\begin{tabular}{lccc}
\hline Energy (kcal) & $1970.08 \pm 666.27$ & $1428.56 \pm 509.87^{*}$ & $1375.46 \pm 496.67^{*}$ \\
kJ & $8248 \pm 2790$ & $5891 \pm 2135$ & $5759 \pm 2080$ \\
Carbohydrate (g) & $196.18 \pm 79.75$ & $135.12 \pm 59.12^{*}$ & $129.49 \pm 57.67^{*}$ \\
Protein (g) & $102.96 \pm 40.66$ & $82.22 \pm 31.54$ & $78.97 \pm 29.05^{*}$ \\
& & & \\
Fat $(\mathrm{g})$ & $76.58 \pm 26.18$ & $57.36 \pm 23.21^{*}$ & $55.40 \pm 22.10^{*}$ \\
\hline
\end{tabular}

593

$594 * \mathrm{p}<0.05$ compared to control

$595 \quad{ }^{a}$ Values are means \pm SD 
$596{ }^{\mathrm{b}} \mathrm{n}=19$

$597{ }^{\mathrm{c}}$ Data analysed using RM-ANOVA

598

599

600

601

602

603

604

605

606

607

608

609

610

611

612

613

614

615

616

617

618

619

620 
621 Table 3: Time until meal request ${ }^{\mathrm{a}, \mathrm{b}, \mathrm{c}, \mathrm{d}}$.

\begin{tabular}{llll}
\hline & Control & CLA & MCT \\
\hline Time (minutes) & $142.11 \pm 42.25$ & $181.58 \pm 61.15^{*}$ & $167.37 \pm 40.50$
\end{tabular}

622

$623 * \mathrm{p}<0.05$ compared to control

$624{ }^{a}$ Data is given in minutes for all tests for total time between the test breakfast and when

625 participants asked for lunch

$626{ }^{\mathrm{b}}$ Values are means $\pm \mathrm{SD}$

$627^{\mathrm{c}} \mathrm{n}=19$

628 d Data analysed using RM-ANOVA

629

630

631

632

633

634

635

636

637

638

639

640

641

642

643 